\title{
General discussion on g-2
}

\author{
Marc Knecht ${ }^{1, a}$ \\ ${ }^{1}$ Centre de Physique Théorique, CNRS/Aix-Marseille Univ./Univ. du Sud Toulon-Var (UMR 7332) \\ CNRS-Luminy Case 907, 13288 Marseille Cedex 9, France
}

\begin{abstract}
Progress made on the theoretical aspects of the standard model contributions to the anomalous magnetic moment of the charged leptons since the first FCCP Workshop on Capri in 2015 is reviewed. Emphasis is in particular given to the various cross-checks that have already become available, or might become available in the future, for several important contributions.
\end{abstract}

\section{Introduction}

The anomalous magnetic moments of the electron $\left(a_{e}\right)$ and of the muon $\left(a_{\mu}\right)$ are among the most precisely measured quantities in particle physics. The latest available experimental results [1-3] read

$$
\begin{aligned}
& a_{e}^{\exp }=1159652180.91(0.26) \cdot 10^{-12}[0.28 \mathrm{ppb}], \\
& a_{\mu}^{\exp }=11659208.9(6.3) \cdot 10^{-10}[0.54 \mathrm{ppm}] .
\end{aligned}
$$

These measurements therefore represent stringent tests of the standard model, and constitute indirect windows towards possible degrees of freedom beyond it. Actually, due to the muon's larger mass, its anomalous magnetic moment is expected to be more sensitive to new physics than the electron's by a factor of $\left(m_{\mu} / m_{e}\right)^{2} \sim 4 \cdot 10^{4}$ [in a strict sense, this argument holds in this simple version provided that i) the new physics is decoupling and that ii) it couples universally to the various lepton flavours]. According to the same argument, the anomalous magnetic moment of the $\tau$ lepton $\left(a_{\tau}\right)$ would even be more sensitive to new physics scales. Unfortunately, the very short lifetime of the $\tau, \tau_{\tau}=290.3(5) \cdot 10^{-15} \mathrm{~s}$, has so far precluded any precision measurement of $a_{\tau}$. The case of the $\tau$ will therefore not be mentioned any further in this review, and for more details on the present status of both experimental and theoretical aspects, the reader is referred to the well-documented existing literature, see for instance Refs. [3-9] and the works quoted therein.

On the theoretical side, the high precision achieved by the experiments has triggered a continuous effort in order to match the level of accuracy of the measurements of $a_{e}$ and $a_{\mu}$. The contributions from quantum electrodynamics (QED) belong to the realm of perturbation theory, and the difficulty here consists in computing all high orders which are relevant at the level of precision displayed in (1). The

\footnotetext{
a e-mail: marc.knecht@cpt.univ-mrs.fr
}

present status achieved in these computations is quite remarkable, since they have been pushed up to the five-loop level. For the weak interactions, the present level of precision is at two loops, with some leading three-loop effects included, which is quite sufficient in view of the present (and future) experimental precision. Finally, contributions from the strong interactions represent the hard core of the theoretical calculations. Here perturbation theory is of little help, since the main contribution comes from the lowenergy regime. Understandably, this is therefore the sector where most recent efforts have gone, and where more progress is needed.

The present review will start from the situation as it was left at, say, the previous Capri Workshop [10] from two years ago, summarizing where progress has been achieved, and trying to point out issues that still need to be improved. For a more general overview, see [11] and references therein.

\section{The present experimental situation}

The value presently predicted by the standard model for $a_{\mu}$ is somewhat lower than the experimental one. This discrepancy now reaches the level of 3.5 standard deviation, and could easily exceed the $5 \sigma$ level, should the new experiments [12-16] confirm the central value in Eq. (1) while reducing its uncertainty by a factor of four. As to the latter point, the first progress made since the workshop held in Capri in 2015 is that there are new data. The commissioning run of the E989 experiment at FNAL collected successfully about 700000 positrons in June 2017. Provided the experiment further runs on schedule, as it did so far, a statistical sample comparable in size to the one collected by the E821 experiment at BNL should become available and be analysed during 2018 [14]. This will already provide a quite valuable experimental cross-check of the value given in Eq. (1). The final target of collecting a statistical sample more than 20 times larger than the 
one collected by the BNL E821 experiment, could then be reached in the year 2020 [14].

Experiment E34 at J-PARC aims at measuring the anomalous magnetic moment of the muon with a comparable improvement in precision, but with a different method, and hence with quite different systematics [16]. Depending on availability of budget and resources, the first results would become available only several years after the planed release of the final results of the FNAL experiment. Nevertheless, since by the year 2020 the anomalous magnetic moment of the muon could well be the only observable showing a deviation from its standard model prediction at a level exceeding 5 standard deviations, this experimental cross-check, although somewhat delayed in time, is highly important and most welcome.

In the case of the electron, the standard model prediction of the anomalous magnetic moment matches the experimental measurement, both in value and in precision:

$$
a_{e}^{\exp }-a_{e}^{\mathrm{SM}}=(-1.30 \pm 0.77) \cdot 10^{-12} .
$$

Notice that an explanation of the $3.5 \sigma$ discrepancy in $a_{\mu}^{\exp }-a_{\mu}^{\mathrm{SM}}$ by new physics scenarios which obey the naive scaling argument described after Eq. (1) would not upset the agreement between $a_{e}^{\exp }$ and $a_{e}^{\mathrm{SM}}$ at the present level of precision.

\section{Progress in the evaluation of the QED contributions}

Three-flavour QED provides more than $99.99 \%$ of the standard-model value of $a_{\mu}$, and it is thus important that the theoretical evaluations are under good control. Here, perturbation theory can be applied $[\ell=e, \mu]$,

$$
a_{\ell}^{\mathrm{QED}}=\sum_{n} C_{\ell}^{(2 n)}\left(\frac{\alpha}{\pi}\right)^{n},
$$

but the challenge lies in the high orders in the powers of $\alpha$, the fine-structure constant, which need to be considered. Up to and including three loops, all contributions are known analytically (references can be found in [17] or [11]), so that the only limitation in precision for second and third orders lies in the precision with which the ratios of the masses of the charged leptons are known. The contributions at fourth and fifth orders are known numerically, and the limitation in precision in these cases comes from the numerical uncertainties in the evaluation of multi-dimensional integrals over Feynman parameters. The present situation reads [18-20]:

$$
\begin{aligned}
& C_{\mu}^{(2)}=1 / 2 \quad C_{\mu}^{(4)}=0.765857425(17) \\
& C_{\mu}^{(6)}=24.05050996(32) \\
& C_{\mu}^{(8)}=130.8780(61) \quad C_{\mu}^{(10)}=750.72(93) .
\end{aligned}
$$

Notice that the uncertainty in $a_{\mu}^{\mathrm{QED}}$ induced by the uncertainties in the values of these coefficients are smaller than those of the present and future experiments. On the other hand, $C_{\mu}^{(8)}(\alpha / \pi)^{4} \sim 3.8 \cdot 10^{-9}$ and $C_{\mu}^{(10)}(\alpha / \pi)^{5} \sim 0.5 \cdot 10^{-10}$, so that at least the value of $C_{\mu}^{(8)}$ should be cross-checked by an independent calculation. This has recently been done $[21,22]$, thanks to the important fact that it is largely dominated by the diagrams containing electron loops, which are enhanced by factors like $\pi^{2} \ln \left(m_{\mu} / m_{e}\right)$. These diagrams can then be evaluated using asymptotic expansion techniques $[23,24]$ in powers (modulo logarithms!) of the small ratio $m_{e} / m_{\mu}$. In this way, all four-loop contributions containing at least one electron and/or tau loop [25] have now been checked, at a level of precision below the one of the value given in Eq. (4), but sufficient to match the level of precision reached by the present experiment value or expected for the future one. The QED prediction for $a_{\mu}$ thus rests on a very safe basis. Adding also the contribution from the weak interactions, $a_{\mu}^{\text {weak }}=15.4(1) \cdot 10^{-10}$, see [26] and references therein, and using the value of $\alpha$ from Ref. [27] [corrected for the recent shift in the value of the Rydberg constant $]$, one obtains $a_{\mu}^{\exp }-a_{\mu}^{\text {QED }}-a_{\mu}^{\text {weak }}=$ $721.65(6.38) \cdot 10^{-10}$.

In the case of the electron, the expansion coefficients read $[17,19,20,28]$ :

$$
\begin{aligned}
& C_{e}^{(2)}=1 / 2 \quad C_{e}^{(4)}=-0.32847844400 \ldots \\
& C_{e}^{(6)}=1.181234016 \ldots \\
& C_{e}^{(8)}=-1.911321390 \ldots \quad C_{e}^{(10)}=6.595(223) .
\end{aligned}
$$

and in several respects the situation looks very different from the muon. First, the contribution to $a_{e}$ from QCD is even much more important than in the case of the muon:

$$
a_{e}^{\mathrm{QED}}=1159652180.277(15)_{\alpha^{5}}(720)_{\alpha(R b 11)} \cdot 10^{-12},
$$

i.e. $a_{e}^{\exp }-a_{e}^{\mathrm{QED}}=0.434(772) \cdot 10^{-12}$, where the error is dominated by the uncertainty in the determination of $\alpha$ [27]. Second, the contributions at order $\alpha^{5}$ is larger than the experimental uncertainty on $a_{e}, C_{e}^{(10)}(\alpha / \pi)^{5} \sim 4.4$. $10^{-13}$, so here its value really matters. Third, the diagrams with loops involving only photons and electrons [the socalled mass-independent contributions] dominate the result, and all diagrams involving muon and/or tau loops are heavily suppressed, by powers of $m_{e} / m_{\mu}$ and $m_{e} / m_{\tau}$. Their values have been computed numerically $[19,20]$ and cross-checked [25] with the asymptotic expansion method. This however leaves out the mass-independent part of the $O\left(\alpha^{4}\right)$ and $O\left(\alpha^{5}\right)$ coefficients, which require an independent cross-check. For the former, such a calculation in semi-analytical form has recently been achieved by S. Laporta $[17,28]$, a quite remarkable tour de force! Only $C_{e}^{(10)}$ remains therefore unchecked so far.

\section{Progress in the evaluation of the hadronic vacuum polarization (HVP)}

The contribution known as hadronic vacuum polarization (HVP) occurs first at order $O\left(\alpha^{2}\right)$, cf. Fig. 1. This lowestorder HVP contribution $\left(a_{\mu}^{\mathrm{HVP}-\mathrm{LO}}\right)$ provides the largest hadronic correction to $a_{\mu}$, but also, to date, one of the largest contribution to the theoretical uncertainty (see Table 1). $a_{\mu}^{\mathrm{HVP}-\mathrm{LO}}$ can be expressed in the following way [29-31]

$$
a_{\ell}^{\mathrm{HVP}-\mathrm{LO}}=\frac{1}{3}\left(\frac{\alpha}{\pi}\right)^{2} \int_{4 M_{\pi}^{2}}^{\infty} \frac{d s}{s} \frac{m_{\ell}^{2}}{s} K\left(m_{\ell}^{2} / s\right) R^{\mathrm{had}}(s),
$$


Table 1. The contributions to $a_{\mu}$ (in units of $10^{-10}$ ) using the latest available values

\begin{tabular}{c|cc}
\hline QED & +11658471.9 & {$[18]$} \\
\hline \hline \multirow{2}{*}{ HVP-LO } & $\left\{\begin{array}{l}+693.1(3.4) \\
+692.23(2.54)\end{array}\right.$ & {$[37]$} \\
& $+688.07(4.14)$ & {$[39]$} \\
\hline \multirow{2}{*}{ HVP-NLO } & $\left\{\begin{array}{l}-9.83(4) \\
-9.93(7)\end{array}\right.$ & {$[38]$} \\
& $\{39]$ \\
\hline \multirow{2}{*}{ HVP-NNLO } & $\left\{\begin{array}{l}+1.24(1) \\
+1.22(1)\end{array}\right.$ & {$[40]$} \\
\hline HLxL & $+10.3(2.9)$ & {$[39]$} \\
\hline EW 1 loop & $+19.48(1)$ & {$[41]$} \\
\hline EW 2 loops & $-4.12(10)$ & {$[26]$} \\
\hline
\end{tabular}

with

$$
K\left(m_{\ell}^{2} / s\right)=\int_{0}^{1} d x \frac{x^{2}(1-x)}{(1-x)+\frac{m_{\ell}^{2}}{s} x^{2}},
$$

and $R^{\text {had }}(s)$ represents the $R$-ratio of the cross section for $e^{+} e^{-} \rightarrow$ hadrons. This contribution can therefore be evaluated using available experimental input. Actually, as illustrated by the second diagram $(b)$ in Fig. 1, what is usually refered to as HVP-LO contains some contributions at order $O\left(\alpha^{3}\right)$, for instance the radiative modes $e^{+} e^{-} \rightarrow \pi^{0} \gamma, \eta \gamma$. The remaining $O(\alpha)\left[O\left(\alpha^{2}\right)\right]$ QED corrections to Fig. 1 are descibed as next-to-leading HVP (HVP-NLO) [nextnext-to-leading HVP (HVP-NNLO)].

Previous data-based evaluations of $a_{\mu}^{\mathrm{LO}-\mathrm{HVP}} \mathrm{had}$ reached a precision around $0.6 \%$ [32-34]. Recently, three new evaluations have been made. Their results, shown in Table 1, are in agreement within the uncertainties given. They include new data (for the experimental aspects, see
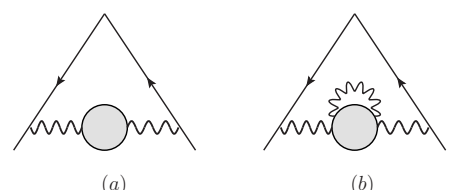

Figure 1. Diagrammatic representation of $a_{\mu}^{\mathrm{HVP}-\mathrm{LO}}$, the leading hadronic vacuum polarization contribution to $a_{\mu}$. In diagram $(a)$, the shaded blob represents the $\langle V V\rangle$ QCD two-point function. Also shown is a next-to-leading contribution, diagram $(b)$, actually included in $a_{\mu}^{\mathrm{HVP}-\mathrm{LO}}$ (see text).

Table 2. The contribution to $a_{\mu}^{\mathrm{HVP}-\mathrm{LO}}$ (in units of $10^{-10}$ ) coming from the measurement of the $e^{+} e^{-} \rightarrow \pi^{+} \pi^{-}$cross section in the region between 600 and $900 \mathrm{MeV}$. Values taken from Fig. 7 in Ref. [42].

\begin{tabular}{l|l} 
Experiment & $a_{\mu}^{\mathrm{HVP}-\mathrm{LO} 2 \pi}(600-900 \mathrm{MeV})$ \\
\hline $\mathrm{BaBar}(09)$ & $376.7(2.7)$ \\
KLOE(comb) & $366.9(2.1)$ \\
$\mathrm{BESIII}(15)$ & $368.2(4.2)$ \\
$\mathrm{SND}(04)$ & $371.7(5.0)$ \\
CMD-2(comb) & $372.4(3.0)$
\end{tabular}

Table 3. Some recent lattice evaluations of $a_{\mu}^{\mathrm{HVP}-\mathrm{LO}}$ (in units of $10^{-10}$ ). The first error is the statistical one, the second the systematic one.

$$
\begin{array}{ll}
654 \pm 32_{-23}^{+21} & {[43]} \\
667 \pm 6 \pm 12 & {[44]} \\
711.0 \pm 7.5 \pm 17.3 & {[45]} \\
715.4 \pm 16.3 \pm 9.2 & {[46]}
\end{array}
$$

$[35,36])$, and reach now a precision around $0.4 \%$. However, some tension remains between the high-precision $e^{+} e^{-} \rightarrow \pi^{+} \pi^{-}$data collected in the region of the $\rho$ resonance, for instance, by BaBar on the one side, and by KLOE and BESIII on the other side, as shown in Table 2 (see also [35]). From a general point of view, given the very high precision achieved, and even more so in view of this tension in the data, some cross-checks would be welcome here also. Several proposal in this direction have been put forward, and are worth investigating.

For one thing, it has been proposed to evaluate the vacuum polarization integral using data not from the timelike region, but from the space-like one, i.e. by considering either Bhabha scattering [48], or muon-electron scattering [49]. While the required statistical accuracy could be reached upon operating for two years at, for instance, the muon beam of $150 \mathrm{GeV}$ available at the CERN North Area, getting the systematic uncertainties below the required level of about $10 \mathrm{ppm}$ constitutes a real challenge. The issue of controling higher-order radiative corrections will also be crucial. The latter aspect has been discussed in several presentations [50-53] at this Worshop, to which I refer the interested reader.

Staying in the Euclidian region, there has been growing interest in the lattice-QCD community to compute $a_{\mu}^{\mathrm{HVP}-\mathrm{LO}}$ through numerical simulations. Several results have appeared since the first FCCP meeting in 2015, and are summarized in Table 3. The precision is still too low, around $2.5 \%$ or more, in order to be competitive with the determinations based on the $e^{+} e^{-} \rightarrow$ hadrons data. Moreover, the systematic uncertainties indicated cover different realities as far as the control over lattice artefacts and/or isospin-breaking effects (see e.g. [47]) is concerned. Nevertheless, the situation looks promising, and one might also consider, as done by the authors of Ref. [46], replacing experimental data by lattice data in the energy regions where the latter are more accurate. Supplementing lattice data for very short and very long distances with experimental measurements of $R^{\text {had }}(s)$, the value shown in the last entry of Table 3 becomes $a_{\mu}^{\mathrm{HVP}-\mathrm{LO}}=692.5(1.4)(0.5)(0.7)(2.1) \cdot 10^{-10}$, where the two first errors come from the lattice, the two last ones from experiment. Combining all these uncertainties in quadrature leads to an overall relative error of less than $0.4 \%$ !

A third approach to the evaluation of $a_{\mu}^{\mathrm{HVP}-\mathrm{LO}}$ has been proposed in Refs. [54-56]. In order to illustrate it, let us come back to Eq. (7), and denote as $f(x ; \kappa)$, with $\kappa \equiv m_{\ell}^{2} / s$ and $0<\kappa \leq \kappa_{0} \equiv m_{\ell}^{2} / 4 M_{\pi}^{2}$, the integrand of the function 
$K(\kappa)$ in (8). From the simple inequalities

$$
f\left(x ; \kappa_{0}\right)<f(x ; \kappa)<\kappa x^{2}\left[1-\kappa x^{2}(1-x)\right],
$$

valid within the ranges of variation of both $x$ and $\kappa$, one obtains the bounds (keeping only the first term on the righthand side of the second inequality reproduces the upper bound obtained long ago in Ref. [57])

$$
\frac{\alpha}{\pi} K\left(\kappa_{0}\right) \mathcal{M}(0)<a_{\ell}^{\mathrm{HVP}-\mathrm{LO}}<\frac{\alpha}{\pi} \frac{1}{3}\left[\mathcal{M}(0)-\frac{11}{10} \mathcal{M}(-1)\right],
$$

in terms of the moments of the Mellin-Barnes transform of the imaginary part of the vacuum polarization function [54],

$$
\mathcal{M}(y)=\int_{4 M_{\pi}^{2}}^{\infty} \frac{d s}{s}\left(\frac{m_{\ell}^{2}}{s}\right)^{y-1} \frac{1}{\pi} \operatorname{Im} \Pi(s), \frac{1}{\pi} \operatorname{Im} \Pi=\frac{1}{3} \frac{\alpha}{\pi} R^{\mathrm{had}} .
$$

Using, for illustrative purposes, the simple toy model for $R^{\text {had }}(s)$ given in Ref. [58], one finds, in the case of the electron, very stringent lower and upper bounds, which in both cases deviate from the actual value given by the toy model by less than $0.1 \%$. The situation in less favourable in the case of the muon. The lower bound amounts to about only $70 \%$ of the actual value, and the upper bound exceeds it by $12 \%$. In order to move beyond the simple bounds of the type shown above requires to use more elaborate mathematical techniques. This has been done by the authors of Refs. [54-56], who obtain a rapidly converging representation in terms of a series of the type

$$
a_{\mu}^{\mathrm{HVP}-\mathrm{LO}}=\sum_{n \geq 0}\left[c_{n} \mathcal{M}(-n)+c_{n}^{\prime} \mathcal{M}^{\prime}(-n)\right]
$$

involving not only $\mathcal{M}(s)$, but also its derivative $\mathcal{M}^{\prime}(s)$. Only a few terms of this series can already provide an evaluation that differs from the full result by less that $1 \%$. The corresponding moments can be determined from phenomenology [59], or computed on the lattice [60].

\section{Progress in the evaluation of the hadronic light-by-light (HLxL)}

Hadronic light-by-light scattering is the next important hadronic contribution to $a_{\mu}$. It involves the fourth-rank vacuum polarization tensor, as shown on Fig. 2. In contrast to the two-point hadronic vacuum polarization tensor involved in $a_{\mu}^{\mathrm{HVP}-\mathrm{LO}}$, there is, in the case of $a_{\mu}^{\mathrm{HLxL}}$, no simple and direct link to an experimental observable, similar to Eq. (7). Other strategies must then be devised in order to overcome the difficulties in handling strong-interaction effects in the non-perturbative regime, while keeping the amount of model dependence at the lowest possible level. Two approaches that can potentially fulfill this condition have been developped in order to evaluate $a_{\mu}^{\mathrm{HLxL}}$ : dispersion relations and numerical simulations of QCD on the lattice.

The dispersive framework set up in Refs. [61, 62] uses a decomposition of the fourth-rank vacuum polarization

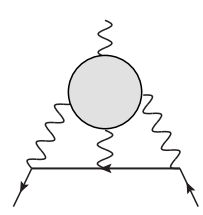

Figure 2. The hadronic light-by-light contribution to $a_{\mu}$. The blob represents the hadronic fourth-rank polarization tensor, i.e. vaccun expectation value of the time-ordered product of four hadronic electromagnetic currents.

tensor into independent invariant functions free of kinematic zeroes and of singularities, for which dispersion relations can be written. These dispersion relations can then be saturated by one-, two- or more meson states and expressed in terms of corresponding form factors. The latter can furthermore be obtained from experiment $[63,64]$ or again... from lattice QCD [65, 66]. Applying this formalism to the $\pi \pi$ intermediate state [67] [68] provides not only a new evaluation of the so-called pion box contribution (see also Ref. [69] for a recent discussion of this contribution), but includes also $\mathrm{S}$-wave rescattering effects. The result obtained this way is very accurate, at the $4 \%$ level. Results concerning other contributions are to be expected in the future. What is so far missing in this dispersive approach, is how QCD short-distance properties [70] (see also the discussion of this issue in [11]) of the fourthrank vacuum polarization tensor will eventually be implemented in this formalism, that is how the various individual contributions will be put together in order to reproduce the correct high-energy behaviour.

Two groups are at present actively involved in the computation of $a_{\mu}^{\mathrm{HLxL}}$ from lattice QCD [71-75]. The authors of Ref. [72] obtain $a_{\mu}^{\mathrm{HLxL}}=5.35(1.35) \cdot 10^{-10}$, where the error is statistical only (finite-volume effects are discussed in Ref. [73]). Although the central value lies somewhat on the low side as compared to current phenomenological estimates $[34,76]$, this new result opens promising prospects for the future.

\section{Conclusion}

The Fermilab g-2 experiment will very soon measure the anomalous magnetic moment of the muon with a precision comparable to the Brookhaven experiment, and, within a couple of years will reduce its uncertainty by a factor or four. Hopefully, the J-PARC experiment E34, which is based on a completely different set-up, will later on provide a cross-check of this important measurement.

QED provides, by far, the largest contribution to $a_{\mu}$, and even more so to $a_{e}$. All numerical evaluations of the contributions at fourth order have now been satisfactorily cross-checked by analytical tools, including the massindependent one, so crucial for $a_{e}$. The only potential problem could come, in the case of the electron, from the fifth-order contribution, which has only been determined numerically, and by one group only. 
The data-based determinations of the contribution to $a_{\mu}$ from hadronic vacuum polarization have been improved thanks to new high-precision experimental input, and more data should become available in the near future, hopefully helping in removing some tensions between different experiments. Cross-checks coming from different approaches seem to be possible. Extracting the vacuum polarization function from $\mu e$ scattering constitutes an interesting proposal in this direction, although the control of the systematic effects represents a quite challenging issue. Interesting developments have also come from numerical simulations of QCD on the lattice. Improvements in the statistical precision and increased control over systematic effects are still needed in order to compete with the databased determinations. Of particular interest is the possibility to combine data and lattice results, taking advantage of the performance of each in various energy regions.

Progress in the determination of the HLxL contribution has been made both in the dispersive approach, and in lattice QCD. The dispersive approach offers the possibility to make use of experimental data or lattice results for various form factors. The results obtained so far are encouraging, and confirm that no important physical effect has been overlooked by the existing phenomenological evaluations. The issue here will rather consist in improving on the present precision and in obtaining reliable estimate of the uncertainties due to lattice artefacts.

\section{Acknowledgements}

I would like to thank the organizers of the Workshop "Flavour changing and conserving processes 2017" for the kind invitation to participate to this second FCCP Workshop, and for choosing the perfect place for such a meeting. This work has been partially supported by the OCEVU Labex (ANR-11-LABX-0060) and the A*MIDEX project (ANR-11-IDEX-0001-02) funded by the "Investissements d'Avenir" French government program managed by the ANR.

\section{References}

[1] D. Hanneke et al., Phys. Rev. Lett. 100, 120801 (2008).

[2] G. W. Bennett et al., Phys. Rev D 73, 072003 (2006).

[3] C. Patrignani et al. [Particle Data Group], Chin. Phys. C 40, 100001 (2016).

[4] G.A. González-Sprinberg et al., Nucl. Phys. B 582, 3 (2000).

[5] S. Narison, Phys. Lett. B 513, 53 (2001); Err. B 526, 414 (2002)

[6] J. Abdallah et al. [DELPHI Coll.], Eur. Phys. J. C 35, 158 (2004).

[7] S. Eidelman, M. Passera, Mod. Phys. Lett. A 22, 159 (2007).

[8] J. Bernabéu et al., Nucl. Phys. B 790, 160 (2008).

[9] S. Eidelman et al., (2016).

[10] G. D’Ambrosio, M. Iacovacci, M. Passera, G. Venanzoni, P. Massarotti and S. Mastroianni, EPJ Web Conf. 118 (2016).
[11] M. Knecht, EPJ Web Conf. 118, 01017 (2016).

[12] R. M. Carey et al.. The New g-2 experiment - 2009. Fermilab. Proposal 0989.

[13] B. Lee Roberts, Chin. Phys. C 34, 741 (2010).

[14] See the contribution of T. Gorringe to this workshop.

[15] T. Mibe [J-PARC g-2 Collaboration], Chin. Phys. C 34, 745 (2010).

[16] See the contribution of G. Marshall to this workshop.

[17] See the contribution of S. Laporta to this workshop.

[18] T. Aoyama et al, Phys. Rev. Lett. 109, 111808 (2012) [arXiv:1205.5370 [hep-ph]].

[19] T. Aoyama et al, Prog. Theor. Exp. Phys. 2012, 01A107 (2012).

[20] T. Aoyama et al, Phys. Rev. D 91, 033006 (2015); Phys. Rev. D 96, 019901(E) (2017).

[21] A. Kurz et al., Phys. Rev. D 92, no. 7, 073019 (2015) [arXiv:1508.00901 [hep-ph]].

[22] A. Kurz et al., Phys. Rev. D 93, no. 5, 053017 (2016) [arXiv:1602.02785 [hep-ph]].

[23] M. Beneke and V. A. Smirnov, Nucl. Phys. B 522, 321 (1998) [hep-ph/9711391].

[24] V. A. Smirnov, Springer Tracts Mod. Phys. 177, 1 (2002).

[25] A. Kurz et al., Nucl. Phys. B 879, 1 (2014) [arXiv:1311.2471 [hep-ph]].

[26] C. Gnendiger et al., Phys. Rev. D 88, 053005 (2013) [arXiv:1306.5546 [hep-ph]].

[27] R. Bouchendira et al., Phys. Rev. Lett. 106, 080801 (2011).

[28] S. Laporta, Phys. Lett. B 772, 232 (2017).

[29] C. Bouchiat and L. Michel, J. Phys. Radium 22, 121 (1961).

[30] L. Durand, Phys. Rev. 128, 441 (1962); Err.-ibid. 129, 2835 (1963).

[31] M. Gourdin and E. de Rafael, Nucl. Phys. B 10, 667 (1969).

[32] M. Davier et al., Eur. Phys. J. C 71, 1515 (2011) [Eur. Phys. J. C 72, 1874 (2012)] [arXiv:1010.4180 [hep$\mathrm{ph}]]$.

[33] K. Hagiwara et al., J. Phys. G 38, 085003 (2011) [arXiv:1105.3149 [hep-ph]].

[34] F. Jegerlehner and R. Szafron, Eur. Phys. J. C 71, 1632 (2011) [arXiv:1101.2872 [hep-ph]].

[35] See the contribution of F. Ignatov to this workshop.

[36] See the contribution of A. Denig to this workshop.

[37] M. Davier et al., Eur. Phys. J. C 77, 827 (2017) [arXiv:1706.09436 [hep-ph]].

[38] See the contribution of D. Nomura to this workshop.

[39] F. Jegerlehner, EPJ Web Conf. 166, 00022 (2018) [arXiv:1705.00263 [hep-ph]].

[40] A. Kurz et al., Phys. Lett. B 734, 144 (2014) [arXiv:1403.6400 [hep-ph]].

[41] R. Jackiw and S. Weinberg, Phys. Rev. D 5, 2396 (1972); I. Bars and M. Yoshimura, Phys. Rev. D 6, 374 (1972); K. Fujikawa et al., Phys. Rev. D 6, 2923 (1972); G. Altarelli et al., Phys. Lett. B 40, 415 (1972); W. A. Bardeen et al., Nucl. Phys. B 46, 315 (1972). 
[42] A. Anastasi et al. [KLOE-2 Collaboration], arXiv:1711.03085 [hep-ex].

[43] M. Della Morte et al., JHEP 1710, 020 (2017) [arXiv:1705.01775 [hep-lat]].

[44] B. Chakraborty et al. [HPQCD coll.], Phys. Rev. D 96, no. 3, 034516 (2017) [arXiv:1601.03071 [hep-lat]].

[45] S. Borsanyi et al. [Budapest-Marseille-Wuppertal coll.], arXiv:1711.04980 [hep-lat].

[46] T. Blum et al., arXiv:1801.07224 [hep-lat].

[47] P. Boyle et al., arXiv:1710.07190 [hep-lat].

[48] C. M. Carloni Calame et al., Phys. Lett. B 746, 325 (2015) [arXiv:1504.02228 [hep-ph]].

[49] G. Abbiendi et al., Eur. Phys. J. C 77, 139 (2017) [arXiv:1609.08987 [hep-ex]].

[50] See the contribution of L. Trentadue to this workshop.

[51] See the contribution of U. Marconi to this workshop.

[52] See the contribution of P. Mastrolia to this workshop.

[53] See the contribution of F. Piccinini to this workshop.

[54] E. de Rafael, Phys. Lett. B 736, 522 (2014) [arXiv:1406.4671 [hep-lat]].

[55] E. de Rafael, Phys. Rev. D 96, no. 1, 014510 (2017) [arXiv:1702.06783 [hep-ph]].

[56] J. Charles et al., arXiv:1712.02202 [hep-ph].

[57] J. S. Bell and E. de Rafael, Nucl. Phys. B 11, 611 (1969).

[58] D. Bernecker and H. B. Meyer, Eur. Phys. J. A 47, 148 (2011) [arXiv:1107.4388 [hep-lat]].

[59] M. Benayoun et al, arXiv:1605.04474 [hep-ph].

[60] S. Borsanyi et al., Phys. Rev. D 96, 074507 (2017) [arXiv:1612.02364 [hep-lat]].
[61] G. Colangelo et al.,JHEP 1409, 091 (2014) [arXiv:1402.7081 [hep-ph]].

[62] G. Colangelo et al., JHEP 1509, 074 (2015) [arXiv:1506.01386 [hep-ph]].

[63] G. Colangelo et al., Phys. Lett. B 738, 6 (2014) [arXiv:1408.2517 [hep-ph]].

[64] A. Nyffeler, Phys. Rev. D 94, no. 5, 053006 (2016) doi:10.1103/PhysRevD.94.053006 [arXiv:1602.03398 [hep-ph]].

[65] X. Feng et al., Phys. Rev. D 91, no. 5, 054504 (2015) [arXiv:1412.6319 [hep-lat]].

[66] A. Gérardin et al., Phys. Rev. D 94, no. 7, 074507 (2016) [arXiv:1607.08174 [hep-lat]].

[67] G. Colangelo et al., Phys. Rev. Lett. 118, no. 23, 232001 (2017) [arXiv:1701.06554 [hep-ph]].

[68] G. Colangelo et al., JHEP 1704, 161 (2017) [arXiv:1702.07347 [hep-ph]].

[69] J. Bijnens and J. Relefors, JHEP 1609, 113 (2016) [arXiv:1608.01454 [hep-ph]].

[70] K. Melnikov and A. Vainshtein, Phys. Rev. D 70, 113006 (2004).

[71] J. Green et al., PoS LATTICE 2015, 109 (2016) [arXiv:1510.08384 [hep-lat]].

[72] T. Blum et al, Phys. Rev. Lett. 118, 022005 (2017) [arXiv:1610.04603 [hep-lat]].

[73] T. Blum et al., Phys. Rev. D 96, 034515 (2017) [arXiv: 1705.01067 [hep-lat]].

[74] See the contribution of C. Lehner to this workshop.

[75] See the contribution of A. Nyffeler to this workshop.

[76] See the contribution of J. Bijnens to this workshop. 